\title{
Towards Developing “Oops! Solver” for Elderly Care
}

\author{
Satoshi NISHIMURA ${ }^{\mathrm{a}, 1}$, Chiaki OSHIYAMA ${ }^{\mathrm{a}}$ and Yuichi OOTA ${ }^{\mathrm{a}}$ \\ ${ }^{a}$ National Institute of Advanced Industrial Science and Technology (AIST)
}

\begin{abstract}
Long-term care costs, burdens of caregivers, and their resultant shortage are prevalent concerns in an increasingly aging society. Use of information and communication technologies is one possible solution to expedite human resource development; however, there are few knowledge resources that are interpretable by computers. In this study, we present Example of structured manuals for elderly care as a knowledge resource for question-answering systems. We conducted semistructured interviews with caregivers in a care facility and retrieved information from an open data set to collect "Oops" incidents in daily caregiving. Based on the collected incidents, we created questions worded in natural language and corresponding computer-interpretable queries. Consequently, we obtained 150 Oops incidents and 33 computer-interpretable queries and confirmed the queries can retrieve relevant knowledge from the knowledge resource.
\end{abstract}

Keywords. Nursing information systems, knowledge bases, geriatric nursing

\section{Introduction}

Medical treatment and nursing costs in Japan are increasing with the progressively aging society. The medical nursing care cost in 2014 was around 950 billion USD, which is about twice of the amount in 1998 [1]. The situation has led to an increasing demand for caregivers. The Ministry of Health, Labour and Welfare estimated a shortage of 377,000 caregivers in 2025 [2].

Past studies have established human resource development as a social problem. Forsell et al. [3] reported on the importance and practice of evidence-based oral health education in geriatric care facilities. The importance of operation management in health care has been underlined in the knowledge management domain [4].

Educational support using information and communication technologies is a possible solution to promote human resource development. Computer-interpretable guidelines are an emerging topic as knowledge bases for clinical decision support systems [5]. In homecare, the heart failure guideline was used by translating a computerinterpretable format [6]. However, there are few reports on usage of guidelines for human resource development because it is difficult for end users, who are unfamiliar with computers, to retrieve knowledge by using computer-interpretable queries.

To aid understanding of the field, a contest called Question Answering over Linked Data is held for end users to encourage them to access data in the Semantic Web domain [7]. This contest is a competition to rewrite a question originally in natural language into

${ }^{1}$ Corresponding Author, Satoshi Nishimura, National Institute of Advanced Industrial Science and Technology (AIST), 2-4-7 Aomi Koto-ku, Tokyo, Japan; E-mail: satoshi.nishimura@aist.go.jp. 
a computer-interpretable query. Such an approach could be applicable to the elderly care domain to facilitate computer-aided learning support.

In this paper, we present the Example of structured manuals for elderly care as a knowledge resource described in a computer-interpretable format in the elderly care domain. We also construct a question-answering data set for Oops solving in this domain. We defined Oops incidents as near-miss incidents or unintended events in daily care, such as "slid off the sliding board" and "forgot to bring ointment after bathing." The data set includes Oops incidents, questions for solving them, and computer-interpretable queries to retrieve knowledge from the knowledge resource.

\section{Methods}

Figure 1 shows an overview of this study. It involved the following steps:

A. Collection of Oops incidents in the elderly care domain.

A1. Collection of Oops incidents from care facility.

A2. Collection of Oops incidents from artificial incident cases available in public.

B. Query creation for Oops solving.

C. Knowledge retrieval from knowledge resource.

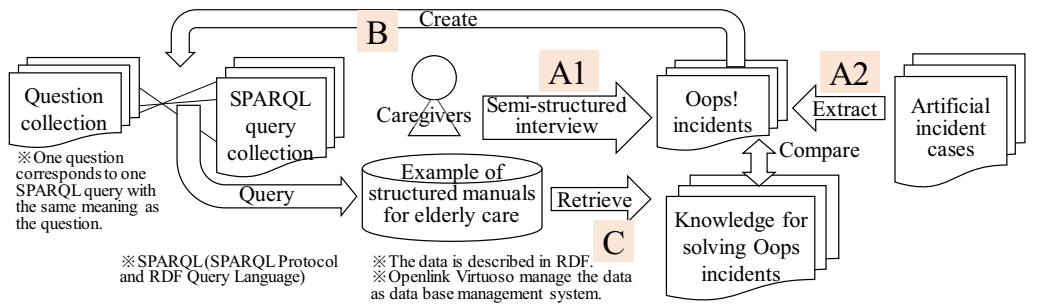

Figure 1. Overview of research flow: first, we collected Oops incidents in the elderly care domain. A1: We collected the incident information from caregivers by semi-structured interview. A2: We extracted the incident information from artificial incident cases which is available to the public. Second, we created natural language question to solve the incidents and corresponding computer-interpretable queries at Step B. Third, we retrieved helpful information from the knowledge base and evaluated the result at Step C.

\subsection{Knowledge Resource: Example of Structured Manuals for Elderly Care}

The Example of structured manuals for elderly care is a knowledge resource. They were extracted, and systematized information related to elderly care actions extracted from five textbooks. We constructed eight structured manuals [8] based on the Convincing Human Action Rationalized Mode (CHARM) [9] notion. A part of the result is depicted in Figure 2. A rounded rectangle node shows an action that is interpreted as a goal. For example, the topmost goal of this process is "Support to move up the slope." To achieve this goal, there are two ways denoted by curved lines. The left part of the figure shows how a care worker interprets the action "an elderly person goes up the slope" as another goal. The action is decomposed into four fine-grained actions described in the lower left of Figure 2. The knowledge resource is formalized in Resource Description Framework $(\mathrm{RDF})^{2}$ and published as open data $^{3}$.

\footnotetext{
${ }^{2}$ RDF 1.1 primer, https://www.w3.org/TR/rdf11-primer/

${ }^{3} \mathrm{https}$ :/github.com/satoshinishimura2460/Example_of_structured_manuals_for_elderly_care
} 


\subsection{Collection of Oops Incidents from Care Facility (A1) and Artificial Incident Cases} (A2)

We collected Oops incidents through semi-structured interviews with caregivers on the challenges faced in daily care work. The interview items are divided into three phases: preparation before care, process during care, and post-care process; for example, "Have you had an experience in which you forgot items in preparation for bathing assistance and had to get it later?" We provide concrete information including type of care with the question so that it becomes easier for caregivers to remember their experiences.

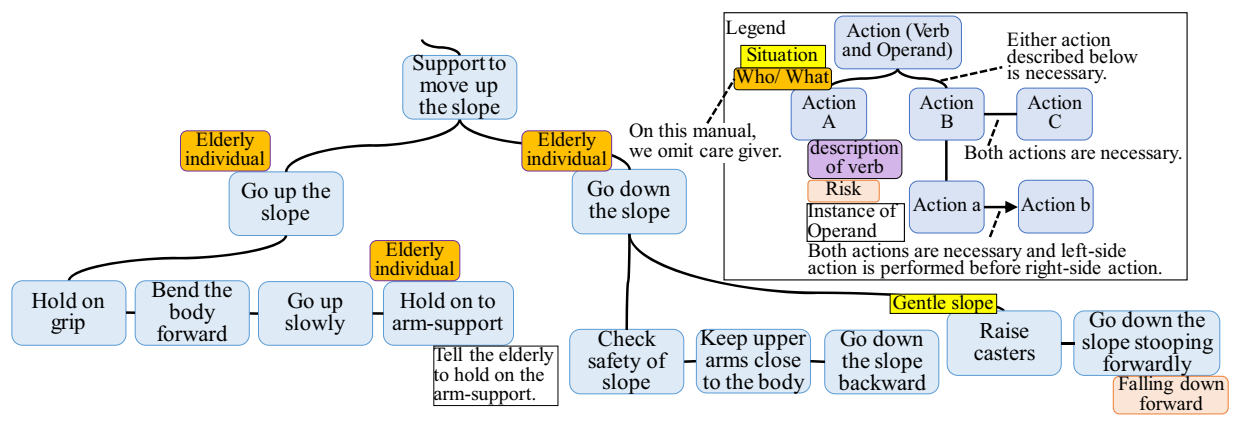

Figure 2. Example of procedural knowledge: Moving support at slope with wheelchair.

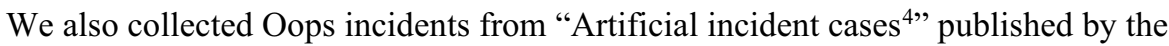
Association for Technical Aids. It is an open data set of artificial examples of incidents based on real incident reports. The number of cases as of August 2019 was 361 . We narrowed down the cases from the following viewpoints of incidents that: occurred during care, harmed care receiver, and occurred during the eight types of care outlined in the knowledge resource.

\subsection{Query Creation for Oops Solving (B) and Knowledge Retrieval from Knowledge Resource (C)}

The queries are aimed at retrieving information to solve the Oops situation collected in the previous section. First, we checked whether the knowledge to solve the situation is included in the knowledge resource. We created questions worded in natural language to retrieve this knowledge from the resource. We created computer-interpretable queries based on the questions worded in natural language. For example, for the Oops situation "slid off the sliding board and was nearly injured," we created the natural language question "What are the risks of using a sliding board?" A corresponding computerinterpretable query was then created to extract relevant knowledge. The extracted knowledge contains "When sitting shallowly to use a sliding board, there is a risk of falling forward." The computer-interpretable query is formalized in SPARQL ${ }^{5}$, which is a web-standard querying language for RDF knowledge base.

Finally, we obtained knowledge retrieval results from the knowledge resource using the created queries. We used Openlink Virtuoso [10] as the database management system, which searches the knowledge resource and interprets the queries.

\footnotetext{
${ }^{4}$ Artificial incident cases (in Japanese), http://www.techno-aids.or.jp/hiyari/

${ }^{5}$ SPARQL 1.1 Query Language, https:/www.w3.org/TR/sparql11-query/
} 


\section{Results}

Through semi-structured interviews with five caregivers, Oops incidents experienced by them were collected. The interviews were held in August 2019 and took 17 to 25 minutes per person. A total of 52 Oops incidents were obtained and 32 of them were related to the eight types of care actions available in the Example of structured manuals for elderly care (knowledge resource). The information extracted from artificial incident cases were narrowed down to 134 cases that occurred during nursing care and 118 cases that occurred during the eight types of care are obtained. The results are shown in Table 1. We obtained 150 Oops incidents related to the eight types of care.

We classified the 150 Oops incidents as solvable or unsolvable based on the knowledge resource. Based on the result, 33 Oops incidents were classified as solvable. We created questions worded in natural language and computer-interpretable queries for the 33 Oops incidents. The questions worded in natural language were created carefully to solve each Oops situation by obtaining knowledge extracted using the question. SPARQL queries were created carefully to convey the same meaning as the corresponding questions worded in natural language. We retrieved knowledge using the created queries. All the 33 queries were effective in retrieving relevant knowledge to solve the Oops situations.

Table 1. Collected Oops incidents.

\begin{tabular}{|l|r|l||l|r|}
\hline & $\begin{array}{l}\text { Facility A } \\
\text { (Total) }\end{array}$ & $\begin{array}{l}\text { Artificial } \\
\text { incidents (Total) }\end{array}$ & $\begin{array}{l}\text { Facility A } \\
\text { (solvable incidents) }\end{array}$ & $\begin{array}{l}\text { Artificial incidents } \\
\text { (solvable incidents) }\end{array}$ \\
\hline Transfer support & 3 & 27 & 3 & 4 \\
\hline Move support & 4 & 48 & 0 & 14 \\
\hline Oral care & 3 & 0 & 0 & 0 \\
\hline Dressing support & 3 & 1 & 1 & 0 \\
\hline Meal assistance & 8 & 2 & 3 & 0 \\
\hline Position change & 4 & 19 & 0 & 3 \\
\hline Bathing support & 3 & 17 & 1 & 2 \\
\hline Excretion support & 4 & 4 & 2 & 0 \\
\hline Total & 32 & 118 & 10 & 23 \\
\hline
\end{tabular}

\section{Discussion}

For each Oops incident, we classified information types necessary for resolving Oops situations. The types of information required were action, risk, detailed information of objects, application conditions of a certain procedure, action attributes, and those not classified in any of them (Other). This classification is based on the schema of the Example of structured manuals for elderly care.

The number of incidents classified as Other was 19, and it was under $20 \%$ of unsolvable incidents. This indicates that the schema of the knowledge resource covers over $80 \%$ of necessary information types for Oops solving.

In addition, it was established that information about action and risk is important to solve Oops incidents. In total, 94 incidents needed action information and 35 needed risk information to arrive at a solution. Of these, 26 incidents that needed action information and 4 incidents that needed risk information were deemed solvable using the knowledge resource. A detailed analysis revealed that incidents related to handling care equipment 
were classified as unsolvable incidents. In other words, the constructed data set for Oops solving revealed a lack of fine-grained information about handling care equipment in the Example of structured manuals for elderly care because it was constructed from general textbooks.

Such data set for Oops solving will serve as the basis for question answering system to provide meaningful information in elderly care domain. Human-computer interaction technology will make them more convenient for elderly care facilities.

\section{Conclusions}

In this paper, we presented a trial to construct a data set for solving Oops situations in the elderly care domain. The data set contained Oops incidents, questions worded in natural language for solving the situation, and computer-interpretable queries. We collected Oops incidents through semi-structured interviews with caregivers at a care facility and open data collection. The questions and queries were constructed based on our proposed knowledge resource. From the result, we obtained 150 Oops incidents, of which 33 were classified as solvable based on the knowledge resource. We believe the data set is a step toward developing a question-answering system for training caregivers.

Throughout the process of query creation and knowledge retrieval, we clarified the lack of information in the knowledge resource. It indicates that the data set also contributes to evaluating the extent of knowledge in the resource for caregiver training.

\section{Acknowledgement}

Part of this work is based on results obtained from a project commissioned by the New Energy and Industrial Technology Development Organization (NEDO).

\section{References}

[1] Ministry of Health, Labour and Welfare. Results of survey of long-term care benefit expenditures for 2016. https://www.mhlw.go.jp/english/database/db-hss/dl/soltcbe2016_b.pdf (2016)

[2] Ministry of Health, Labour and Welfare. Estimation of supply and demand of caregivers in 2025. https://www.mhlw.go.jp/file/04-Houdouhappyou-12004000-Shakaiengokyoku-ShakaiFukushikibanka/270624houdou.pdf_2.pdf (in Japanese) (2015)

[3] Forsell M, Kullberg E, Hoogstraate J, Johansson O, Sjögren P. An evidence-based oral hygiene education program for nursing staff. Nurse education in practice 11(4) (2011), 256-259.

[4] Svensson A, Hedman E. Knowledge management for operations management within health care. In Proc. of European Conference on Knowledge Management (2018), 847-854.

[5] Peleg M. Computer-interpretable clinical guidelines: a methodological review. Journal of biomedical informatics, 46(4) (2013), 744-763.

[6] Topaz M, Shalom E, Masterson-Creber R, Rhadakrishnan K, Monsen KA, Bowles KH. Developing nursing computer interpretable guidelines: a feasibility study of heart failure guidelines in homecare. In Proc. of AMIA Annual Symposium Proceedings (2013), 1353-1361.

[7] Lopez V, Unger C, Cimiano P, Motta E. Evaluating question answering over linked data. Web Semantics: Science, Services and Agents on the World Wide Web 21 (2013), 3-13.

[8] Nishimura S, Zhao L, Fukuda K, Nishimura T. Building structured manuals for elderly care as a computer interpretable knowledge resource. In Proc. of Joint International Semantic Technology Conference, Poster and Demonstration Paper (2018), 78-81. 
[9] Nishimura S, Kitamura Y, Sasajima M et al. CHARM as activity model to share knowledge and transmit procedural knowledge and its application to nursing guidelines integration. Journal of Advanced Computational Intelligence and Intelligent Informatics 17(2) (2013), 208-220.

[10] Erling O, Mikhailov I. RDF support in the Virtuoso DBMS. Networked Knowledge-Networked Media, Springer, Berlin, Heidelberg, (2009), 7-24. 